\title{
Progressive paraparesis, lymphocytic meningitis, and stroke in a 37-year-old man
}

\author{
J Bamford, J Ironside, H McNaughton, C P Warlow
}

\section{Case presentation}

The patient is a right-handed male, born in 1951 , and is married. He is an electronics solderer.

In January 1989 this man began to feel tired and lethargic and noticed weight loss despite a reasonable appetite. $\mathrm{He}$ also described night sweats, possibly in association with a temperature. By March he was beginning to notice weakness in the right leg, and then the left leg. He developed tingling and numbness in the left foot, which spread up to the knee, and with aching in the left leg. He began to notice pain in the right testicle and right leg; the latter gradually became numb. By May he was hardly able to walk and he developed urinary retention. His health was otherwise good. He did not smoke and drank about three pints ( 1.7 litres) of beer a day. $\mathrm{He}$ previously had had multiple exostoses (familial) removed from both hands. There was no other family history of illness.

On examination in June 1989 he was thin and was noted at times to have mild pyrexia. He had lost about $25 \mathrm{~kg}$ in weight over the previous six months. General examination was otherwise, and remained, normal. On neurological examination, higher functions, cranial nerves and both arms were normal. He was areflexic in the legs and plantar reflexes were bilaterally extensor. Tone was normal. Both legs were globally weak, the right one more than the left. Pain sensation was reduced in both feet and along the inner aspect of the right leg. Joint-position sense was lost in both feet. At this stage a urinary catheter was inserted and he was confined to a wheelchair.

The following initial investigations were found to be normal: full blood count, calcium, phosphate, protein electrophoresis, alkaline phosphatase, thyroid function, glucose, liver function (except $\gamma$-glutamyltransferase $=69$ units/l, normal <42), chest radiograph, ECG, blood cultures, and a full myelogram. The CSF was xanthochromic, however, and protein levels ranged from $3 \cdot 1$ to $7 \cdot 0 \mathrm{~g} / 1$. The immunoglobin $G$ level and index were normal. There were 189 lymphocytes $/ \mathrm{ml}$. The level of glucose in the CSF was $2.6 \mathrm{mmol} / 1$ compared with $6.2 \mathrm{mmol} / \mathrm{l}$ in the blood. Cultures for bacteria, fungi, and tuberculosis were negative on several occasions; India ink stains were also negative. There were no malignant cells.
Further investigations which were found to be normal included: barium meal, upper gastrointestinal endoscopy, brain CT, MRI of the brain and cervical cord, sural nerve biopsy, bone marrow aspiration and trephine, serum amylase, antinuclear factor, rheumatoid factor, lupus anticoagulant, anticardiolipin antibodies, and complement levels, B12 and folate, blood porphyrins, Treponema pallidum haemagglutination assay (TPHA), HIV antibodies, CD4/CD8 ratio, antibodies against toxoplasmosis, mycoplasma, borrelia, Epstein-Barr virus, herpes zoster, herpes simplex, cytomegalovirus, measles, and mumps virus titres, and an ultrasound scan of the abdomen including liver, spleen, and kidneys. Nerve conduction was normal in the arms but in the legs, the left sural sensory action potential was absent and the right was small. There was no conduction in the peroneal nerves. In the legs, the muscles appeared to be slightly denervated on EMG. A muscle biopsy was non-specifically abnormal, however, with no definite evidence of denervation. There was selective type II fibre atrophy.

By the end of June 1989 the numbness and weakness had extended to his abdomen and he was still occasionally pyrexial (up to $38^{\circ} \mathrm{C}$ ) with night sweats. He was started on corticosteroids and improved somewhat neurologically but developed pressure sores. Azathioprine was added and the steroids were gradually reduced in October. Ten days after the steroids were stopped, he awoke to find that he was weak in the left arm and face, and had slurred speech. On examination that day there was dysarthria and an upper motor neuron weakness of the left side of his face. He was weak in the left arm and the left arm reflexes were brisker than the right. In the legs he had no movement at all, absent reflexes, and plantar reflexes were flexor. There was a sensory level at T4 and there was no joint-position sensation in the feet. A CT scan on the next day showed a low-density area lateral to the right lateral ventricle. The CSF protein was still raised at $2 \cdot 3 \mathrm{~g} / 1$ and there were 38 lymphocytes/ $\mu$ l. All CSF cultures were again negative and the blood tests were unremarkable. The haemoglobulin was $11.2 \mathrm{~g} / 1$ and the erythrocyte sedimentation rate was $24 \mathrm{~mm}$ in the first hour. The previous tests were repeated and were again normal. Brain biopsy was declined by the patient. 
Figure 1 Non-contrast axial brain $C T$ scan showing haemorrhagic infarct in the region of the left basal ganglia.

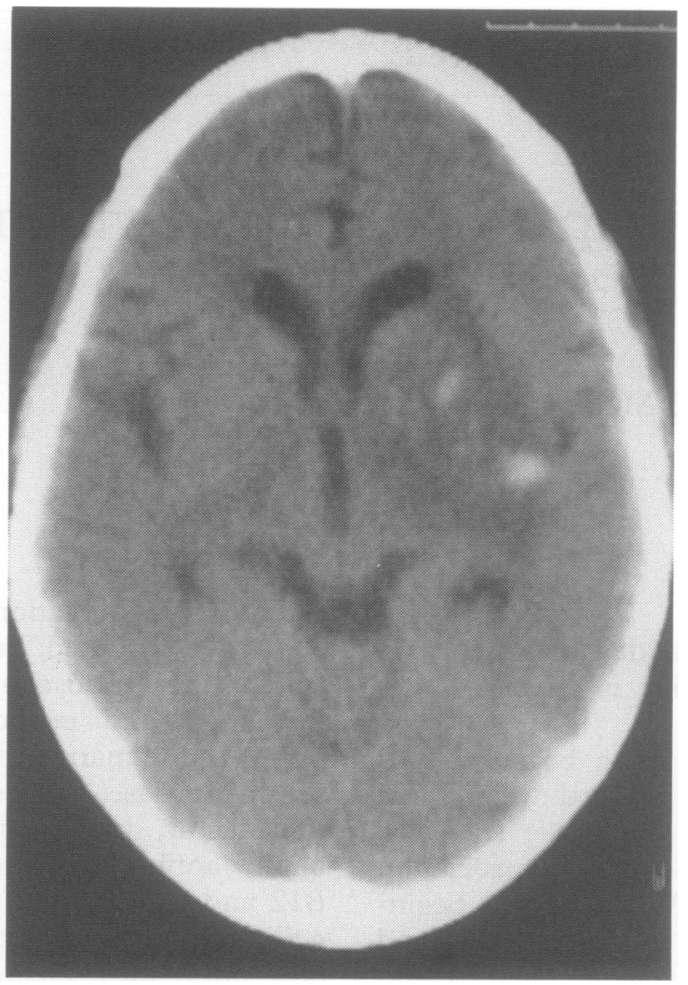

Over the next several months he developed infected pressure sores, occasional chest infections, and urinary infections. He had intermittent pyrexia. In May and June 1990 the corticosteroids were being reduced when, on 17 June, he suddenly became aphasic with weakness of the right arm, possibly with a right homonymous hemianopia. A plain CT scan (fig 1) showed a haemorrhagic infarct in the left basal ganglia. His swallowing was so badly impaired that a nasogastric tube was inserted. An EEG showed widespread slowing bilaterally. The corticosteroids were again increased and there may have been some improvement. He was still completely paralysed in his legs, catheterised, and confined to a wheelchair. He was eventually placed in long-term care, and gradually declined. $\mathrm{He}$ died in January 1992. As far as we know there were no further, overt neurological events, except that, weeks before his death, he had an episode of aphasia and weakness in his right arm. A postmortem examination was performed.

\section{Discussion}

DR JOHN BAMFORD:

This man's initial symptoms are non-specific, but give hints of an inflammatory, infective, or neoplastic aetiology. The neurological deterioration was rapid with the initial symptoms of right leg weakness in March, and yet by the same summer he was paraplegic and catheterised. The presenting symptoms suggest a cauda equina syndrome although there were some unusual features, not the least being the fact that the motor deficit preceded the sensory one. I wonder whether there was central and peripheral disease in the neuraxis early on since the history is of a global weakness in the legs. This, rather than the focal weakness usually associated with cauda equina lesions, suggests cord involvement, albeit that the signs (areflexia and flexor plantars), point to a lower motor neuron problem. Additionally, one notes that proprioception is much more affected than spinothalamic modalities. In vasculitic conditions affecting peripheral nerves, the larger myelinated fibres may be picked out preferentially. The normal myelograms reasonably exclude a mass lesion and I note the absence of thickened or infiltrated nerve roots which might have been a source of a tissue diagnosis, although the radiology can be normal even with extensive lymphomatous infiltration of nerve roots. Finally, the testicular pain might be radicular in origin rather than due to primary testicular pathology.

So we come to the CSF: there is a very high protein content and the immediate assumption would be that the xanthochromia relates to this, although, in view of subsequent events, the possibility of a haemorrhagic pathology needs to be remembered. There is a lymphocyte response and a reduction in the CSF glucose compared with the blood of more than $50 \%$. The differential diagnosis of these abnormalities is extensive, but in the clinical context, tuberculous meningitis (TBM) must be high on the list. Hospitalised patients with TBM have CSF white cell (predominantly lymphocyte) counts of up to $1000 / \mathrm{ml}$ with high protein and low glucose levels. The direct smear is positive for acid-fast bacilli in $10-85 \%$ of cases, depending on technique, with positive cultures in 45-90\%. ${ }^{1}$ The chest radiograph was normal in this case but up to $50 \%$ of patients with TBM have no evidence of tuberculosis elsewhere. ${ }^{1}$ So far, polymerase chain reaction techniques for rapid diagnosis of TBM have been disappointing. The clinician here was faced with the problem of an active CSF compatible with TBM, in a man whose neurological condition was deteriorating rapidly. Furthermore, the use of steroids was being contemplated. There are those clinicians, and I think I may be one of them, who would start treatment with antituberculosis drugs in this situation while awaiting the results of cultures. On the other hand, the normal, contrast-enhanced CT scan, in particular the absence of basal meningeal enhancement or hydrocephalus, would argue against TBM.

Fungal meningitis was obviously looked for with India ink stains on multiple CSF specimens, although I would have preferred to have had the result of the cryptococcal antigen test. ${ }^{2}$

Sarcoidosis clearly merits consideration. Matthews' review ${ }^{3}$ remains the most useful here. There are neurological symptoms in 5\% of patients with sarcoid disease although pathologists find evidence of CNS involvement more frequently. Neurosarcoid may occur without demonstrable involvement elsewhere, and may present in ways that could explain this patient's symptoms. A 
cauda equina syndrome is a recognised presentation of neurosarcoid, although less common than isolated cranial nerve palsies and a granulomatous leptomeningitis. Furthermore, it is capable of giving a mixture of central and peripheral disease. Most of the CSF findings of the case were compatible with neurosarcoid, although one notes that the protein level was originally higher than those quoted by Matthews.

Although uncommon in the UK, brucellosis should be mentioned. There are six types of the small, non-sporing, non-motile, Gram-negative coccobacillus. All but one of these are recognised human pathogens and host specificity is not exclusive. Acute brucellosis rarely presents to neurologists but they should be aware of the distinction between chronic brucellosis and neurobrucellosis. The hallmark is the active CSF. There may be an acute meningoencephalitis, but also a chronic meningitis, with or without a vasculitic or granulomatous component, or both. ${ }^{4}$ In the present case, the three months of lassitude and weight loss at the onset would be compatible with brucellosis, as would the subsequent neurological symptoms and CSF findings.

A comment should be made about the neurophysiology. There are asymmetrical sural potentials of a degree that would be abnormal for a man of this age, and thus support a multifocal disorder rather than a diffuse polyneuropathy. We are not told which muscle was biopsied, and therefore one cannot make too much of the apparent discrepancy between the EMG and the muscle biopsy with respect to denervation. I would also have done a muscle biopsy, although the findings turned out to be rather non-specific: in arteritic conditions, muscle biopsy has been said to have a yield of $35-40 \%$, and granulomas are sometimes found. ${ }^{5}$

At this stage I would have liked to have seen the results of an antineurotrophil cytoplasmic antibody (ANCA) test, and may well have asked for ENT and ophthalmic opinions, thinking of Wegener's granulomatosis and uveitis respectively. It is clear that lymphoma was, quite correctly, repeatedly considered as a diagnostic possibility, but all reasonable tests were negative.

Following treatment with steroids, he had a stroke-like episode involving the left arm and face, with dysarthria. The CT scan shows a low-density area just above the internal capsule on the right, which would be quite compatible with an infarct. The area is too large to be in the territory of a single perforating artery, despite the fact that the clinical details we are given would be compatible with a partial lacunar syndrome (although one cannot be sure that adequate testing of non-dominant cortical function was performed). Thus one is thinking of disorders that might involve multiple striate branches; a vasculitis related to a basal meningitis or an embolus to the proximal middle cerebral artery for example. Marantic endocarditis needs to be considered, especially in view of the subsequent event in the other hemisphere, and I would have requested an echocardiogram, although this may be negative in marantic endocarditis. For what it is worth, there is no hyperdensity in the middle cerebral artery on the nonenhanced CT scan, which would have suggested the presence of acute thrombus.

A CNS vasculitis is looking more likely now. Of the primary vasculitides, polyarteritis nodosa has a predilection for the peripheral nervous system, and a CSF reaction of this order would be very unlikely. Wegener's granulomatosis is a possible candidate although the lack of chest symptoms counts against it. Sarcoid remains a distinct possibility and giant cell arteritis, although unlikely, needs to be considered. Although a lymphocytic response of this magnitude can be seen in the CSF of patients with Behçet's disease, the protein is probably too high, and he would not fulfil the usual diagnostic criteria. Isolated CNS angiitis usually presents with headache and confusion. Secondary vasculitis may be seen with lymphoma, histiocytosis, and hairy cell leukaemia, but none of the other investigations support these diagnoses. Similarly, there is a lack of support for an infection-related vasculitis, such as that following herpes zoster.

The second cerebral event was a haemorrhagic infarct which was larger than the infarct on the right, although probably still within the striate territory. Miller Fisher ${ }^{6}$ suggested that such haemorrhagic change was the hallmark of cardiogenic embolism, with the embolus impacting and causing ischaemia of the distal capillaries followed by fragmentation and the rupture of those same capillaries when re-exposed to systemic blood pressure. More recently, CT studies have suggested that it may be the size of the infarct, rather than the aetiology, that influences this change, and, in at least one case, haemorrhagic change has been demonstrated distal to an occluded artery. Anecdotally, vascultic infarcts are more likely to undergo haemorrhagic change. In the absence of a cardiac lesion, or evidence of degenerative vascular disease, the presence of infarction in both hemispheres points towards a vasculitic process.

I think the diagnosis rests between sarcoidosis and isolated angiitis of the CNS, and I would favour the latter. Although it usually presents with cerebral symptoms, such as change in mentation, headache, and hemiparesis, $20 \%$ present with paraparesis, fever, and weight loss. There are published cases presenting with a fluctuating myelopathy, and one case of a cauda equina syndrome. ${ }^{7}$ As far as the CSF is concerned, a number of cases presenting with a myelopathy had high CSF proteins and a lymphocyte response similar to this case. Intracerebral and subarachnoid haemorrhage occurs (perhaps reflected in the xanthochromic CSF). The condition is variably steroid responsive and the overall clinical pattern of this case would be compatible with the diagnosis. It is the condition par excellence where a leptomeningeal biopsy is indicated. 
Figure 2 The cut surfaces of the cerebrum show a recent haematoma in the right parietal lobe, with old infarcts in the head of the right caudate nucleus (which appears shrunken) and in the globus pallidus, putamen, internal capsule, and insular cortex on the left. A smaller area of haemorrhagic infarction is noted in the left parietal lobe.

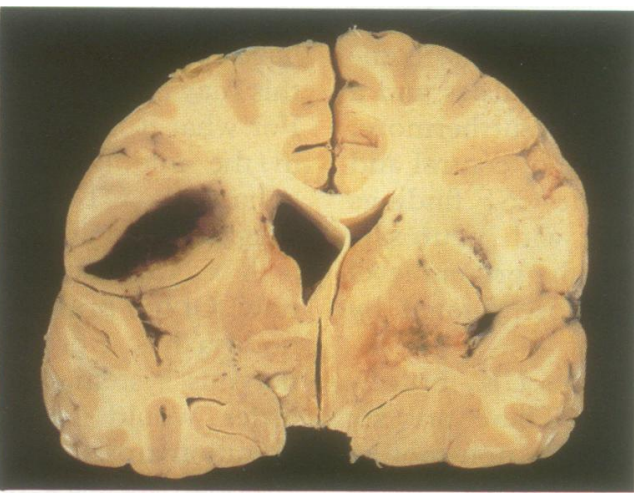

DR JAMES IRONSIDE:

The postmortem examination was performed by a general pathologist in a different town. The brain and spinal cord were sent here for detailed examination.

The major autopsy findings were of bilateral suppurative bronchopneumonia with hilar lymphadenopathy thought to be

$A$

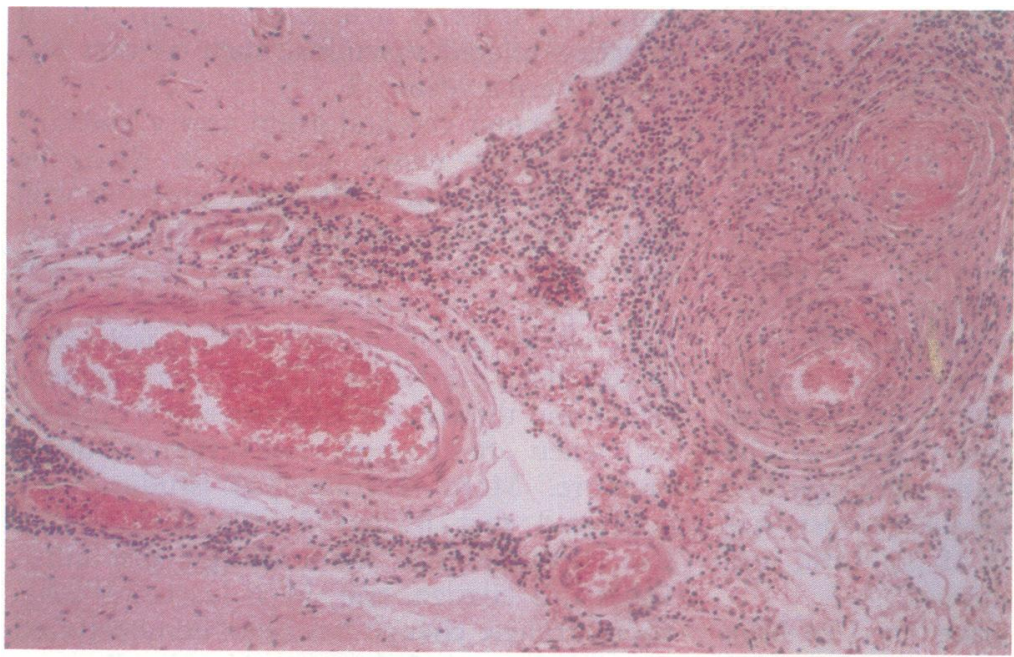

$B$

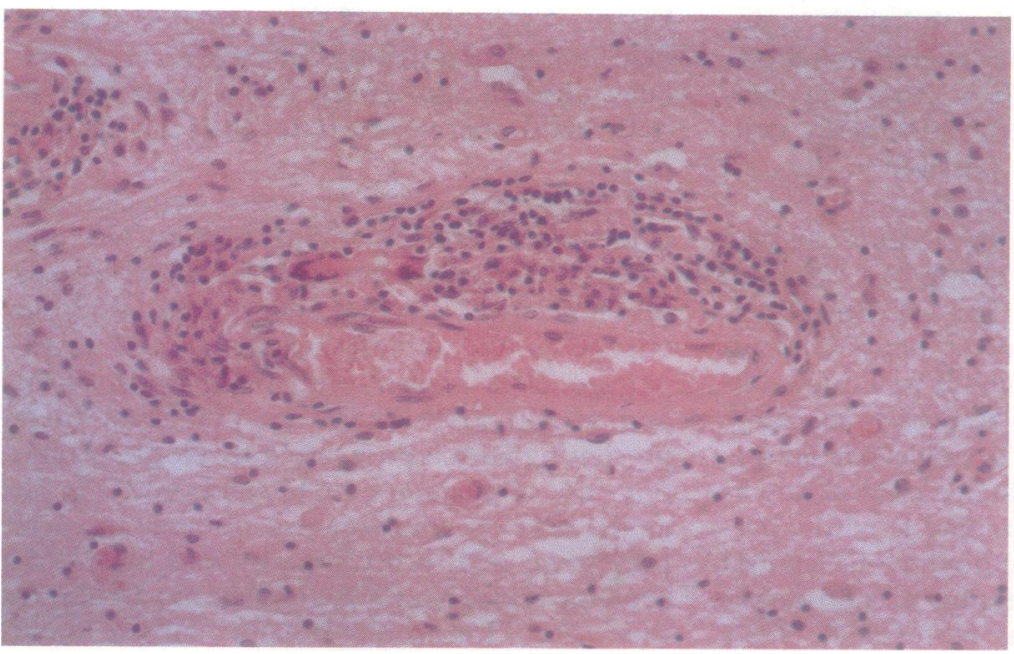

Figure $3(A)$ In the leptomeninges there is a dense perivascular inflammatory infiltrate around small arteries and arterioles (right). These cells comprise lymphocytes, plasma cells and epithelioid macrophages. One of the small arteries shows fibrinoid necrosis with occlusion of the lumen. The veins in this region (left) appear relatively spared. (B) Granulomatous inflammation with multinucleate giant cells is present in the perivascular space around a cerebral arteriole. No evidence of necrosis or caseation is noted. secondary to infection. Cachexia was noted and there were widespread pressure sores. On gross examination of the brain, multiple small foci of haemorrhage within the subarachnoid space were noted. The left occipital lobe showed a greyish discoloration with multiple haemorrhagic areas suggestive of recent infarction. On cross section, a small area of established infarction was noted in the midpons and at several regions in the cerebellar hemispheres. In the cerebral hemispheres, haematomas were present within the parietal lobes bilaterally, and established areas of infarction were noted in the basal ganglia on the left side and in the head of the caudate nucleus on the right (fig 2). Multiple smaller areas of haemorrhagic infarction were noted throughout the cerebrum. The spinal cord was markedly abnormal, being shrunken and discolored and reduced to a ribbon-like strip in the mid-thoracic region. On cross section, there was extensive tissue destruction at this site, and multiple smaller areas of haemorrhage and congestion were noted elsewhere around blood vessels in the cord. Histological examination revealed an inflammatory infiltrate around blood vessels in the meninges and parenchyma, with necrosis of small arteries and occlusion of some vessels (fig 3A). The veins were well preserved and spared by the inflammatory process. The inflammatory cell infiltrate was polyclonal and there were no organisms including fungi. No malignant cells were present. In the brain itself there were collections of cells around blood vessels consisting of epithelioid macrophages. These were by definition granulomas, and contained occasional giant cells (fig 3B). In the spinal cord, the anterior spinal artery was involved by the inflammatory process with granulomatous change. Some of the vessels in the cord were occluded and the cord itself was very damaged. In cross section the midthoracic region was demyelinated with tissue destruction. A section of the cauda equina showed inflammatory change around small vessels and a small granuloma.

There was an acute bronchopneumonia in the lungs. Lymph nodes from the hilum showed large, pink, epithelioid macrophages with no necrosis or caseation although fibrosis was present. Giant cells were present but stains for tuberculosis were negative and there were no inclusions characteristic of sarcoid.

In summary, there was haemorrhage and infarction throughout the CNS, with a granulomatous angiitis. The significance of the lymph node changes in the hilum are uncertain but they are not diagnostic of sarcoid. Other organs were extensively sampled and there was no evidence of granulomas elsewhere including the testes.

\section{Conclusions}

Dr Fohn Bamford's diagnosis: isolated angiitis of the CNS.

Pathological diagnosis: Granulomatous angiitis of the CNS; granulomatous lymphadenitis of hilum. 


\section{Comment}

Whether the granulomatous changes in the hilar lymph nodes were an incidental finding, or connected with the neurological process remains unclear. Granulomatous angiitis of the CNS is probably a non-specific pathological reaction to a number of disease processes. ${ }^{7}$ The differentiation between granulomatous angiitis of the CNS and neurosarcoidosis, for example, may be a matter of definition rather than representing two separate disease processes. ${ }^{8}$

This CPC was presented at the Edinburgh Advanced Neurology course, April 11993.
1 Molavi A, LeFrock JL. Tuberculous meningitis. Med Clin North Am 1985;69:315-31.

2 Sabetta JR, Andriole VT. Cryptococcal infection of the central nervous system. Med Clin North Am 1985; 69:333-44.

3 Matthews WB. Neurosarcoidosis. In: Vinken PJ, Bruyn $\mathrm{GW}$, eds. Handbook of clinical neurology. Amsterdam Elsevier/North Holland; 1979;38:521-42.

4 Bharucha NE, Bhabha SK, Bharucha EP. Infections of the nervous system. In: Bradley WG, Daroff RB, Fenichel GM, Marsden CD, eds. Neurology in clinical practice. Boston: Butterworth-Heinemann 1991; practice.

5 Loughlin M. Muscle biopsy: a laboratory investigation. Oxford: Butterworth-Heinemann 1993;143.

6 Fisher CM, Adams RD. Observations on brain embolism with special reference to the mechanisms of hemorrhagic infarction. $f$ Neuropathol Exp Neurol 1951;10: rhagic

7 Hankey GJ. Isolated angiitis/angiopathy of the central nervous system. Cerebrovasc Dis 1991;1:2-15.

8 Uhrich H. Neurosarcoidosis or granulomatous angiitis. A problem of definition. Mt Sinai f Med 1977;44:718-25. 